\title{
Chapter 3 \\ Computer simulations of the structure of nanoporous carbons and higher density phases of carbon
}

\author{
Lydia Alonso, Julio A. Alonso, and María J. López
}

\begin{abstract}
The most stable form of solid carbon is graphite, a stacking of graphene layers in which the carbon atoms show $s p^{2}$ hybridization which leads to strong intralayer bonding. Diamond is a denser phase, obtained at high pressure. I $\mathrm{n}$ diamond the carbon atoms show $s p^{3}$ hybridization. Metastable solid carbon phases can be prepared also with lower density than graphite (in fact, densities lower than water); for instance the carbide-derived carbons. These are porous materials with a quite disordered structure. Atomistic computer simulations of carbide-derived carbons indicate that the pore walls can be viewed as curved and planar nanographene ribbons with numerous defects and open edges. Consequently, the hybridization of the carbon atoms in the porous carbons is $s p^{2}$. Because of the high porosity and large specific surface area, nanoporous carbons find applications in gas adsorption, batteries and nanocatalysis, among others. We have performed computer simulations, employing large simulation cells and long simulation times, to reveal the details of the structure of the nanoporous carbons. In the dynamical simulations the interactions between the atoms are represented by empirical many-body potentials. We have also investigated the effect of the density on the structure of the disordered carbons and on the hybridization of the carbon atoms. At low densities, typical of the porous carbidederived carbons formed experimentally, the hybridization is $s p^{2}$. On the other hand, as the density of the disordered material increases, a growing fraction of atoms with $s p^{3}$ hybridization appears.
\end{abstract}

Work dedicated to Professor N.H. March

Lydia Alonso · Julio A. Alonso · María J. López

Departamento de Física Teórica, Atómica y Óptica, Universidad de Valladolid, 47011 Valladolid, Spain

e-mail: jaalonso@fta.uva.es,e-mail: maria.lopez@fta.uva.es 


\subsection{Introduction}

Nanoporous carbons exhibit a quite disordered structure with internal pores of nanometric size. These materials have low densities and high specific surface areas, properties which make them attractive in applications to gas adsorption and hydrogen storage, among others. The carbide-derived carbons (CDC) form an interesting family of nanoporous carbons. CDCs can be easily produced from metal carbides [1, 2], $\mathrm{ZrC}$ for instance, by selectively extracting the metal atoms through a chemical chlorination process performed at temperatures between $600{ }^{\circ} \mathrm{C}$ and $1200{ }^{\circ} \mathrm{C}$. The structural characteristics and the properties of these materials can be tuned by selecting the production conditions, in particular the reaction temperature, and the appropriate post-treatments of the samples. The microstructure of the CDCs has been investigated by Raman spectroscopy, X-ray diffraction and high-resolution transmission electron microscopy [3]. The elucidation of the structure of nanoporous carbons is greatly aided by computer simulations. Atomistic computer simulations of carbidederived carbons reveal a disordered structure of connected pores of different size and shape $[4,5]$. The pore walls can be viewed as curved and planar graphene fragments with numerous defects and some open edges. Consequently, the hybridization of the carbon atoms in this family of porous materials is that typical of graphene, that is, $s p^{2}$. The increase of the experimental reaction temperature, and the increase of the temperature and annealing time in the computer simulations, both induce a progressive repair of the long range disorder and a substantial increase of the degree of graphitization in the system [6]. A main characteristic of the porous carbons is their small densities (number of atoms per unit volume), and this is the reason for the easy formation of a network of interconnected planar and curved two dimensional graphene-like layers. In the other extreme, diamond is a solid phase of carbon denser than graphite, produced under extreme high pressure and temperature conditions in the earth mantle or by synthetic methods in the laboratory $[7,8,9]$. The atomic coordination around each carbon atom in diamond is four, and the electronic hybridization is $s p^{3}$. Although diamond is metastable (the most stable solid phase of carbon is graphite), the rate of conversion of diamond to graphite is negligible at ambient conditions.

Early in 1977, Norman March and coworkers pioneered the investigation of the structure of amorphous carbon $[10,11]$. Their conclusion was that the X-ray and electron diffraction intensities cannot be explained by simply considering a model structure and the appropriate atomic scattering factor, and that an explicit modelling of the chemical (covalent) bonds, is required. Amorphous carbon obtained by different experimental methods shows values of the interatomic distances and coordination numbers in between those for graphite and diamond [12, 13]. An early proposal for the structure of amorphous carbons was a disordered mixture of small twodimensional graphitic fragments (in which the carbon atoms have $s p^{2}$ hybridization) linked by tetrahedrally bonded $\left(s p^{3}\right)$ atoms [14]. However, neutron diffraction revealed little tetrahedral bonding [12]. This is easy to understand, because the densities are not in the high density regime favorable to tetrahedral bonding. In fact, our simulations of porous carbons for samples with more than sixty thousand atoms 
[4] revealed disordered structures formed by planar and curved graphitic fragments connected in a way leaving abundant empty spaces (pores), and the analysis of the structures indicated a very small amount of tetrahedrally coordinated carbon, certainly below one per cent. The resulting material can be viewed as low density $(0.77$ $\mathrm{g} / \mathrm{cm}^{-3}$ ) disordered carbon. Despite the highly disordered structure this type of carbons are not clasified as amorphous carbons. According to the recommendations of IUPAC [15], the term amorphous carbon is restricted to carbon materials having short-range order only (no long-range crystalline order) with deviations of the interatomic distances and/or interbonding angles with respect to the graphite lattice as well as to the diamond lattice. So, the term amorphous carbon is not applicable to materials with two-dimensional structural elements such as polyaromatic layers with a nearly ideal interatomic distance and an extension greater than one nanometer. Using density-functional molecular dynamics and simulation cells containing 216 atoms, Deringer and Csanyi [16] have found that the structure of amorphous carbon is a mixture of threefold $\left(s p^{2}\right)$ and fourfold $\left(s p^{3}\right)$ bonded atoms, the proportion of these depending sensitively on the density. The densities investigated by these authors are between 1.5 and $3.5 \mathrm{~g} / \mathrm{cm}^{-3}$ (notice that the densities of graphite and diamond are $2.1-2.2 \mathrm{~g} / \mathrm{cm}^{-3}$ and $3.5 \mathrm{~g} / \mathrm{cm}^{-3}$, respectively). Motivated by all the above works we present here the results of an investigation of the structure of non crystalline carbons (including amorphous carbon) over a wide range of densities, between $0.77 \mathrm{~g} / \mathrm{cm}^{-3}$ and $2.87 \mathrm{~g} / \mathrm{cm}^{-3}$. One of the main purposes is to investigate the transition between disordered porous carbons and amorphous carbons, and to detect the critical density for which the amount of atoms with tetrahedral $\left(s p^{3}\right)$ coordination begins to be noticeable. Because the proportion of atoms with tetrahedral coordination is expected to be small, except at high densities, it is necessary to work with simulation cells having a large enough number of atoms.

\subsection{Theoretical models and dynamical simulations}

Molecular dynamics simulations are performed to investigate the structural features of a variety of disordered carbon materials spanning a broad range of densities $\left(0.7-2.8 \mathrm{~g} / \mathrm{cm}^{3}\right)$. CDCs are porous carbon materials placed on the low density corner of pure carbon materials with densities smaller than that of water. Experimentally, these materials are produced from precursor metal carbides or silicon carbide, by selective chemical extraction (through chlorination) of the metal or silicon atoms. Upon removal of the metal or silicon, the carbon sublattice becomes very unstable and collapses internally. The transformation experienced by the material is a conformal transformation, that is, a transformation without change of the macroscopic piece shape or size, giving rise to the porous carbon structure. To mimic the formation proccess of the CDCs, we start the simulations after the removal of the metal atoms or silicon (the chlorination procedure is out of the scope of the present study) with the $\mathrm{C}$ atoms in the same structure as in the corresponding carbides, and perform molecular dynamics simulations at constant number of particles 
$N$, constant volume $V$, and constant temperature $T$, at several temperatures. Thus we simulate the structural transformation of the carbon skeleton of the carbide keeping constant the density of the carbon material along the simulation, similarly to the experimental conformal transformation. Two carbides have been considered: $\mathrm{ZrC}$, which has a structure of two fcc interpenetrated lattices, and $\alpha$-SiC, which has a $6 \mathrm{H}$-hexagonal structure. The densities of the $\mathrm{C}$ atoms in the carbides are $0.77 \mathrm{~g} / \mathrm{cm}^{3}$ and $0.96 \mathrm{~g} / \mathrm{cm}^{3}$, respectively. Large simulation cells are used within the periodic boundary conditions scheme to represent appropriately the disordered CDC porous structures. A $12 \times 12 \times 12$ cubic cell of $56.376 \AA$ of side, containing $6912 \mathrm{C}$ atoms is used to simulate the $\mathrm{CDC}$ derived from $\mathrm{ZrC}$, and a $10 \times 6 \times 18$ tetrahedral cell of dimensions $53.36 \times 55.458 \times 45.3 \AA$, containing $6480 \mathrm{C}$ atoms is used for the CDCs derived from SiC. Amorphous carbons, on the other hand, are disordered high density carbons, with densities between the density of graphite $2.1-2.2 \mathrm{~g} / \mathrm{cm}^{3}$ and the density of diamond, $3.5 \mathrm{~g} / \mathrm{cm}^{3}$. As the density of carbon increases from the densities typical of CDCs to the densities typical of amorphous carbons, the structure of the material is expected to experience a profound transformation. To assess the dependence of the final carbon structures on the density and on the initial configuration of the carbon atoms we have considered two types of materials, M1 and M2, having the initial structure of the carbon squeleton in $\mathrm{ZrC}$ and $\mathrm{SiC}$, respectively, but with scaled $\mathrm{C}-\mathrm{C}$ distances to produce the desired densities in the range of $0.77-2.87 \mathrm{~g} / \mathrm{cm}^{3}$. Notice that the CDC derived from $\mathrm{ZrC}$ is the $\mathrm{M} 1$ material with density $0.77 \mathrm{~g} / \mathrm{cm}^{3}$ and the CDC derived from $\mathrm{SiC}$ is the $\mathrm{M} 2$ material with density $0.96 \mathrm{~g} / \mathrm{cm}^{3}$.

The selected temperatures for the simulations ${ }^{1}, T=350,2100$ and $3010 \mathrm{~K}$, are higher than the temperatures of the chlorination process of about $600-1500 \mathrm{~K}$. The reason being that the former determine the speed of the structural changes in the material whereas the latter correspond to the chemical procedure for extraction of the metal or silicon [4]. Moreover the simulation time, of the order of a few hundred picoseconds, is orders of magnitud smaller than the experimental times of several minutes or hours. Thus, the higher temperatures of the simulations spead up the structural changes and therefore compensate for the shorter simulation times. An empirical correlation can be established between the temperatures of the simulations and the experimental ones $[4,17]$. To start the simulations, the initial velocities of the carbon atoms are set to a Maxwellian distribution corresponding to the selected simulation temperature. The Nosé-Hoover thermostat is used to keep constant the temperature along the simulations. The equations of motion are integrated with a time step of $0.5 \mathrm{fs}$. Simulation runs of $300 \mathrm{ps}$ lead to well converged final structures, as it has been checked by performing simulation runs of $600 \mathrm{ps}$ for some selected cases. The final structures obtained in the simulations are quenched down to $0 \mathrm{~K}$ in order to perform the structural analysis. The structures are then characterized on the basis of (i) the local ordering as measured by the coordination of the $\mathrm{C}$ atoms, which reflect the $s p^{2}$ or $s p^{3}$ hybridization of $\mathrm{C}$, and the ring pattern of the $\mathrm{C}$ network, and (ii) global quantities such as the specific surface area of the pore walls, the porosity

\footnotetext{
${ }^{1}$ Notice that the Tersoff potential gives a melting temperature for carbon of about $6000 \mathrm{~K}$ whereas the experimental value is about $4300 \mathrm{~K}$. Therefore the temperatures given in this paper have been scaled by a 0.7 factor.
} 


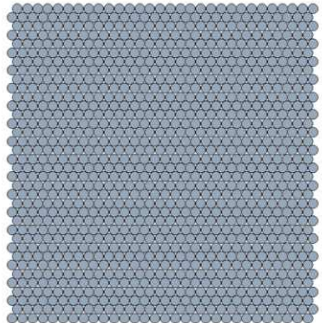

$\mathrm{t}=\mathbf{0} \mathrm{ps}$

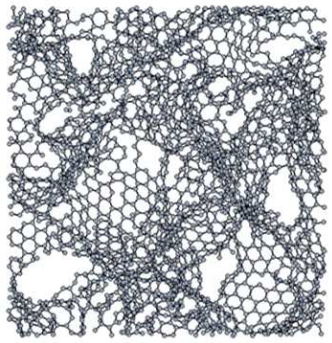

$\mathrm{t}=150 \mathrm{ps}$

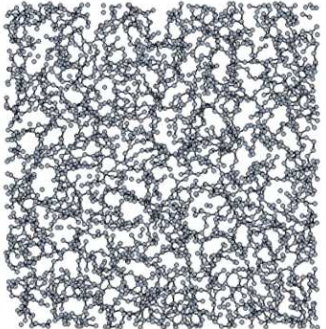

$\mathrm{t}=0.5 \mathrm{ps}$

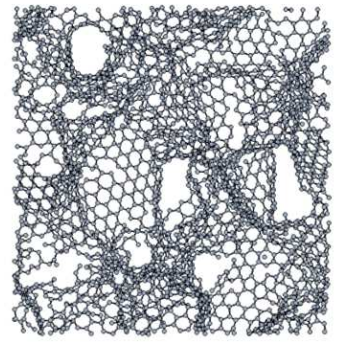

$\mathrm{t}=\mathbf{3 0 0} \mathrm{ps}$

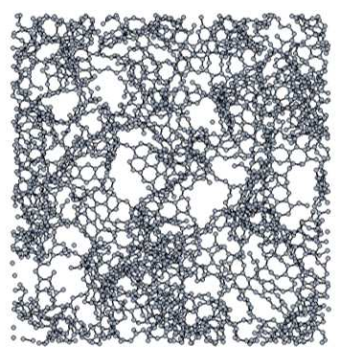

$t=5 p s$

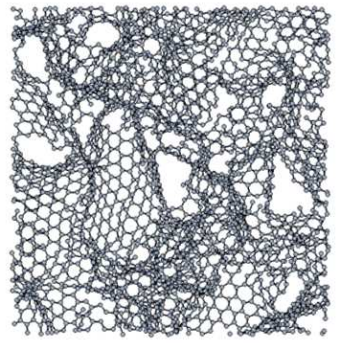

$t=600$ ps

Fig. 3.1 Snapshots, for several times, of the formation of the $\mathrm{CDC}$ structure derived from $\mathrm{SiC}$ at a simulation temperature of $3010 \mathrm{~K}$. A slab of $25 \AA$ of depth is shown for clarity.

(ratio of empty to total volume) and the size distribution of pores. In the simulations, the interatomic $\mathrm{C}-\mathrm{C}$ interactions are mimicked through the Tersoff potential $[18,19,20]$, that appropriately represents the covalent bonds between carbon atoms in graphite and diamond, plus a weak interaction potential [21] which is added to represent the interaction between layers in graphite.

\subsection{Results}

We start investigating the formation of CDCs derived from $\mathrm{ZrC}$ and $\mathrm{SiC}$ through NVT molecular dynamics simulations. The chemical (clhorination) procedure used to extract the metal from the carbides is not simulated here. The simulation starts just after the removal of the metal atoms from the carbide and before the carbon atoms had time to move. Thus, the initial structure for the simulations consists in a carbon skeleton in which the carbon atoms are placed at the same positions that they occupied in the corresponding carbide lattice. The NVT molecular dynamics is aimed to simulate the subsequent conformal trasformation experienced by the carbon network. Thus, the density of the carbon material remains fixed in the simulations and is equal to the density of the carbon atoms in the original carbide. 

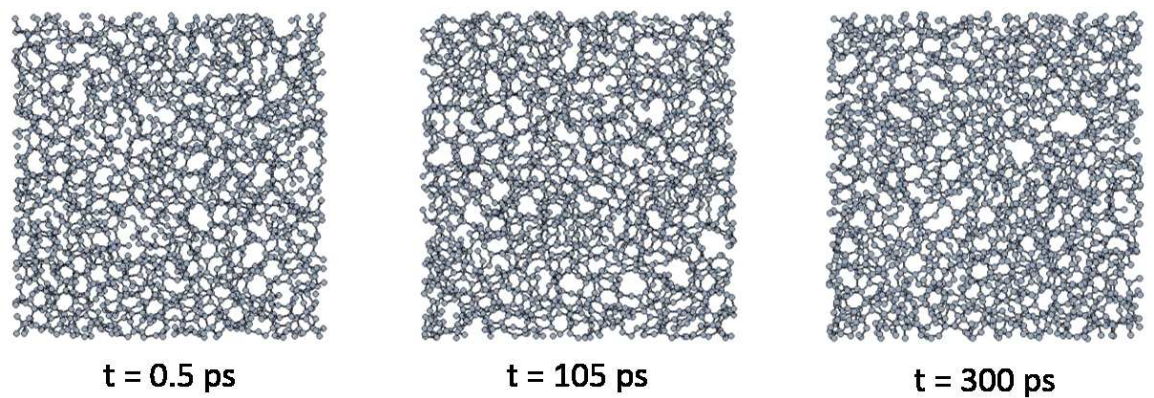

Fig. 3.2 Snapshots, for several times, of the formation of the M1 material with a density of $2.2 \mathrm{~g} / \mathrm{cm}^{3}$, at a simulation temperature of $3010 \mathrm{~K}$. A slab of $10 \AA$ of depth is shown for clarity.

Clearly, after the removal of the metal atoms from the carbide the structure of the carbon atoms is highly unstable, and the internal collapse of the structure occurs very fast. $\mathrm{C}-\mathrm{C}$ bonds begin to form in the material, and after $0.5 \mathrm{ps}$ most atoms are linked together forming a highly disordered carbon network. Figure 3.1 shows several snapshots at different times of the simulation of the formation of a CDC derived from $\mathrm{SiC}$ at a simulation temperature of $3010 \mathrm{~K}$. After about $5 \mathrm{ps}$, the structure evolves forming an incipient porous structure which fully develops at longer times. After $300 \mathrm{ps}$, the final porous structure is almost reached, the pore walls are graphitic layers with defects, interconnected with each other. It is clearly apparent that the carbon atoms are not uniformly distributed in the material. Longer simulation times of the order of $600 \mathrm{ps}$ only lead to minor modifications of the nanoporous structure of the CDC. This can be explained by the high thermal stability of the graphitic nanostrips [22]. Quite similar formation pathways and final structures have been obtained for CDCs derived from the other precursor carbide, $\mathrm{ZrC}$, at the same $(T=3010 \mathrm{~K})$ simulation temperature [4].

However, different structures are produced for carbon materials with higher densities, of the order or higher than the density of graphite. As an example, Fig. 3.2 shows snapshots along the dynamics, of the formation of the M1 material (initial fcc configuration of the $\mathrm{C}$ atoms) with a density of $2.2 \mathrm{~g} / \mathrm{cm}^{3}$. The internal collapse of the carbon structure also occurs very fast, in about $0.5 \mathrm{ps}$, as in the previous case. However, at this relatively high density, the subsequent evolution of the carbon network is quite different. In contrast with the CDC's, the high density M1 material does not develop a porous structure and it is not possible to identify in the structure flakes of graphitic layers. The $\mathrm{C}$ atoms form, instead, a disordered three-dimensional $\mathrm{C}$ network.

The local structure of the carbon network and the level of graphitization of the pore walls can be analized by investigating the coordination of the carbon atoms. The coordination number is defined as the number of nearest neighbors of an atom. Three-fold coordinated carbon atoms with $s p^{2}$ hybridization are characteristic of two-dimensional graphitic layers. The edges of finite size graphitic flakes give rise to the appearance of two-fold coordinated atoms. On the other hand, coordination 


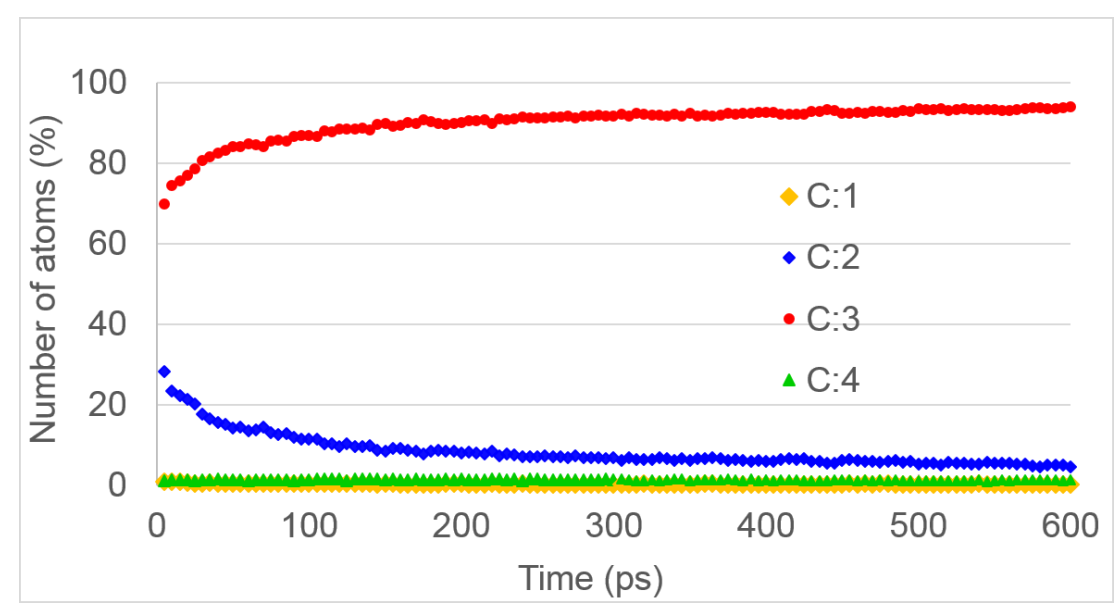

Fig. 3.3 Ratio of carbon atoms with coordinations one, two, three and four as a function of the simulation time, for the $\mathrm{CDC}$ structure derived from $\mathrm{SiC}$ at a simulation temperature of $3010 \mathrm{~K}$.

four $\left(s p^{3}\right.$ hybridization) leads to three dimensional structures and is characteristic of diamond. Figure 3.3 shows the time evolution along the simulation of the coordinations of the $\mathrm{C}$ atoms in the $\mathrm{CDC}$ structure obtained from the $\mathrm{SiC}$ precursor at a temperature of $3010 \mathrm{~K}$. Coordinations two an three rise up within a few picoseconds. In about $5 \mathrm{ps}, 70 \%$ of the $\mathrm{C}$ atoms become three-fold coordinated and about $30 \%$ become two fold coordinated. Coordinations one and four, on the other hand, are only marginaly present in the structure. As the time proceeds, the number of threefold coordinated atoms increases slowly at the expense of the two-fold coordinated atoms. This indicates an increase of the size of the graphitic flakes accompained by the corresponding reduction of the edges. After about $600 \mathrm{ps}$ the structure does not change any further and the different coordinations become almost flat as a function of time. The ratio of the number of $\mathrm{C}$ atoms with different coordinations is well converged within $1 \%$. A slightly relaxed convergence criterion of $2-3 \%$ is fulfilled with simulation times of about 300 ps (see Fig. 3.3). A similar convergence with time has been found for the simulated M1 and M2 structures generated at different temperatures and for different densities of carbon. Therefore, we will limit the simulations to $300 \mathrm{ps}$ in order to reduce the computational cost of this study. The final structures obtained in the simulations are then quenched down $0 \mathrm{~K}$ in order to perform the structural analysis of the materials at equilibrium conditions.

For the low density materials (M1 and M2 with densities below $1.7 \mathrm{~g} / \mathrm{cm}^{3}$ ), the number of three-fold coordinated atoms increases with the simulation temperature indicating that the level of graphitization of the structure improves with temperature. However, even at the highest simulation temperature $(T=3010 \mathrm{~K})$, the twofold coordinated atoms do not disappear completely; this shows the imposibility of removing all the edges of the graphitic flakes and therefore the impossibility of fully graphitize the sample. As a representative example, the left panel of Fig. 3.4 shows 

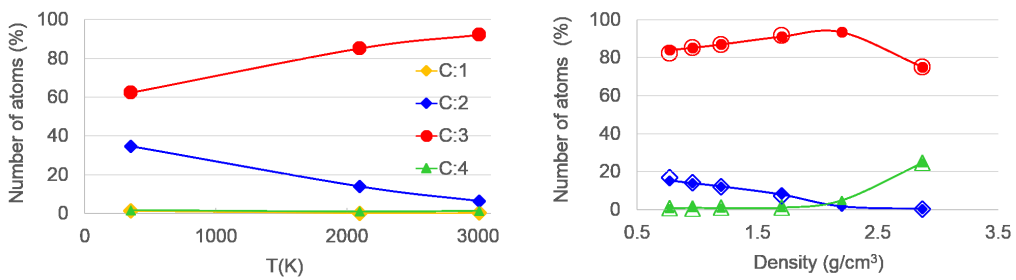

Fig. 3.4 Number (in percent) of carbon atoms with coordinations one, two, three and four. Left pannel gives the coordinations as a function of the simulation temperature for the CDCs derived from SiC. Right pannel gives the coordinations as a function of the density for the M1 (open symbols) and M2 (solid symbols) materials produced at $T=2100 \mathrm{~K}$.

the coordinations as a function of temperature of the CDCs produced using $\mathrm{SiC}$ as the precursor.

Figure 3.4 also shows, in the right panel, the coordinations of the $\mathrm{C}$ atoms in the $\mathrm{M} 1$ and M2 materials as a function of the $\mathrm{C}$ density. The number of three-fold coordinated atoms increases and the number of two-fold coordinated atoms decreases with increasing density, up to approximately the density of graphite $\left(2.2 \mathrm{~g} / \mathrm{cm}^{3}\right)$. This increase of 3-fold coordinated atoms, however, is not indicative of an improvement in the level of graphitization of the material, as can be clearly seen from the snapshots of Fig. 3.2. The graphitic layers become smaller and more interconnected among them giving rise to a highly disordered three-dimensional network of $s p^{2}$ $\mathrm{C}$ atoms. For larger densities, above the density of graphite, the trend changes and the number of three-fold coordinated atoms decreases in favor four-fold coordinated atoms, with $s p^{3}$ hybridization, which are characteristic of diamond-like structures. Thus, the materials with a density of $2.7 \mathrm{~g} / \mathrm{cm}^{3}$ are formed by a mixture of threeand four-fold coordinated $\mathrm{C}$ atoms with a ratio of about three $(75 \%)$ to one $(25 \%)$, respectively. This mixture of coordinations is also found in amorphous carbon materials [16] although one should notice that a broad range of densities and ratio of coordinations is included under the generic name of amorphous carbons. The structures obtained for a given density and at a given simulation temperature for the two types of materials investigated here, M1 and M2, are very similar, what leads us to conclude that the initial configuration of the $\mathrm{C}$ atoms for the simulations (cubic or hexagonal) has little effect in the final structure of the simulated materials. The more relevant parameter being the density of carbon atoms.

The ring pattern of the carbon network is also used to characterize the structure of the simulated materials. In a perfect graphitic layer the carbon atoms are threefold coordinated and form a honeycumb network of hexagonal rings. The presence of pentagonal rings in graphitic layers gives rise to closed carbon structures as in the fullerenes, whereas heptagonal and octagonal rings lead to open structures as in schwarzites. On the other hand, non planar hexagonal rings are also found in diamond like structures but, in this case, the $\mathrm{C}$ atoms form a non-layered threedimensional network of four-fold coordinated atoms. At the low carbon densities of the $\mathrm{CDCs}$ derived from $\mathrm{ZrC}$ and from $\mathrm{SiC}$, the walls of the porous structure of these 

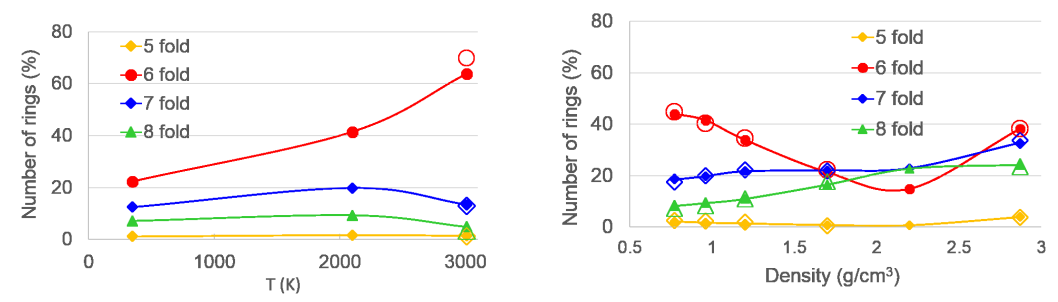

Fig. 3.5 Number (in percent with respect to the ideal number of hexagonal rings in a graphitic layer) of five-, six-, seven-, and eight-fold rings. Left pannel: number of rings as a function of the simulation temperature for the CDCs derived from SiC. The solid and empty symbols correspond to simulation times of 300 and 600 ps, respectively. Right pannel: number of rings as a function of the density for the M1 (open symbols) and M2 (solid symbols) materials produced at $T=2100 \mathrm{~K}$.

materials are mainly formed by hexagonal rings, defining graphitic layers. Pentagonal and smaller rings are completely absent and, therefore, closed pores can not be formed. However, there is a non negligible number of heptagons and octagons in the layers which lead to the interconnection of the pore walls and to the open character of the pores in these materials. The number of hexagonal rings increases with increasing simulation temperature, as it is shown in Fig. 3.5 (left panel) for the CDCs derived from $\mathrm{SiC}$ (a similar behaviour is observed for the CDCs derived from $\mathrm{ZrC}$ ). This confirms the observed improvement with temperature of the level of graphitization of the pore walls. However, the number of heptagons and octagons, although smaller than the number of hexagons, remains almost constant with temperature; these rings do not disappear even at the highest simulation temperature, what prevents the formation of large graphitic layers.

The right pannel of Fig. 3.5 reveals that with increasing carbon density, up to approximately the density of graphite, the number of hexagons decreases whereas the number of heptagons and octagons increases. As a consequence, the graphitic structure and the local order of the structure disappear and, as it is observed in Fig. 3.2, a three-dimensional disordered network of $s p^{2} \mathrm{C}$ atoms develops. For densities above the density of graphite, the number of hexagonal rings increases again and the number of heptagons and octagons also increases. The hexagonal rings, however, now contain four-fold coordinated carbon atoms and are the signature of the development of diamond like structures. Thus, at high densities (below the densidy of diamond, $3.53 \mathrm{~g} / \mathrm{cm}^{3}$ ) the materials become amorphous with highly disordered structures and formed by a mixture of three- and four-fold coordinated $\mathrm{C}$ atoms, as discussed above.

A relevant quantity to characterize nanoporous materials is the specific surface area (SSA), which it is correlated, in general, with the capacity of the material to adsorb gases. The geometrical evaluation of this quantity from the simulated structures is quite difficult, because the walls of the pores are formed by small, non planar layers interconnected among them. Thus, it has been proposed [4] to approximate the SSA by the sum of the areas of the rings each considered as a perfect planar ring. Although the pore walls are one layer thick, the two sides of the walls are not 


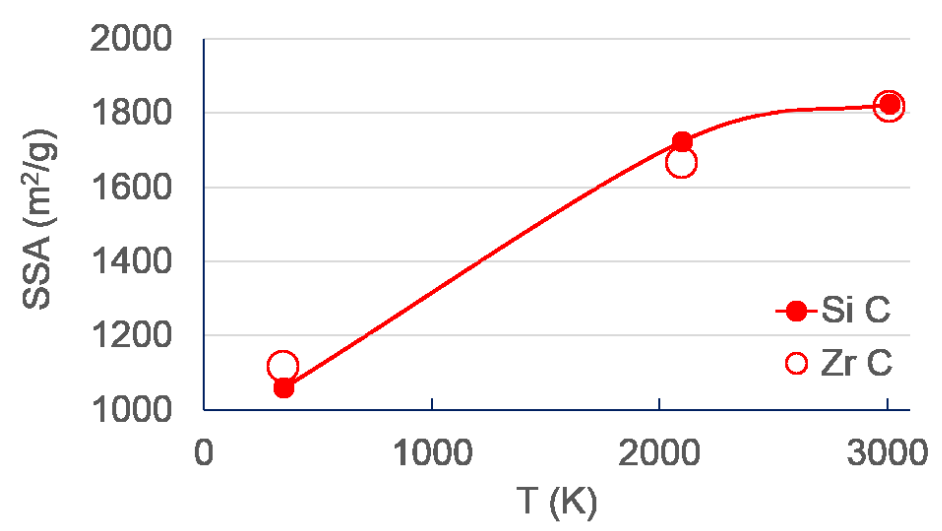

Fig. 3.6 Specific surface area (SSA) as a function of the simulation temperature for the CDCs derived from $\mathrm{ZrC}$, empty symbols, and from $\mathrm{SiC}$ solid symbols.

fully available for gas adsorption, mainly due to the interconnection between layers. From the simulated structures one can estimate that, in average, only about one side and half of the other side of the layers are exposed for gas adsorption. Thus to calculate the SSA, we multiply the surface of one side of the layers by the empirical factor of 1.5. Figure 3.6 shows the SSA for the CDCs derived from $\mathrm{ZrC}$ and $\mathrm{SiC}$ as a function of temperature. The SSA increases moderately with the temperature of the simulation following a similar trend as the experimentally determined SSA [23] for various $\mathrm{CDCs}$ as a function of chlorination temperature. The simulated values match rather well the experimental values. Moreover we found no much difference between the two low density CDC's investigated, derived from $\mathrm{ZrC}$ and from $\mathrm{SiC}$, respectively. For the high density simulated materials, the geometrical determination of the SSA is not physically meaningful since the number of rings does not correlate with the surface available for gas adsorption, as it has been seen above.

Of great interest is the porosity of the materials, that is, the empty volume that can be used to adsorb molecules or for catalysis. We have calculated the porosity from the simulated geometrical structures, considering an effective exclusion volume (volume that can not be used to adsorbe gases) of each $\mathrm{C}$ atom given by a sphere of radius equal to the Van der Waals radius of carbon $(1.7 \AA)$. The porosity increases weakly with the simulation temperature, as it is shown in Fig. 3.7. The porosity of the $\mathrm{CDCs}$ derived from $\mathrm{ZrC}$ is larger than that determined for $\mathrm{SiC}$, due to the density difference between the two materials (the CDCs derived from $\mathrm{ZrC}$ have a lower density). However calculated values of the porosity for the $\mathrm{CDCs}$ derived from $\mathrm{ZrC}$ are slightly smaller than the ones obtained in previous simulations [4]. This is due to the smaller simulation cells used here. Since the porosity is a global quantity of the material, large cells are required for an accurate determination of the porosity although the trends with temperature and density can be already revealed with smaller simulation cells as the ones used here. Moreover, the experimentally determined values 

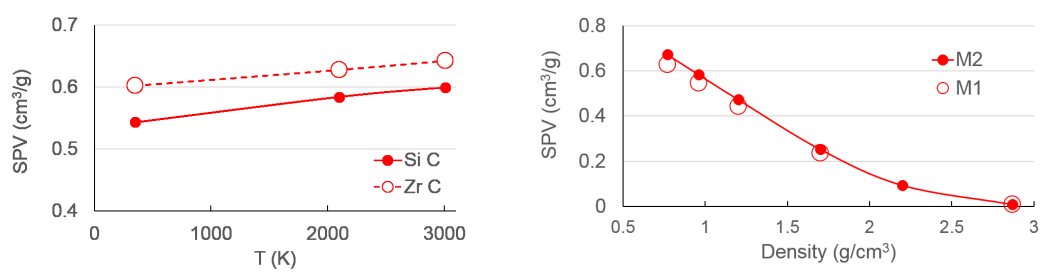

Fig. 3.7 Specific pore volume, SPV. Left pannel: SPV as a function of the simulation temperature for the CDCs derived from $\mathrm{ZrC}$, empty symbols, an from SiC solid symbols. Right pannel: SPV as a function of the density for the M1 (open symbols) and M2 (solid symbols) materials produced at $T=2100 \mathrm{~K}$

exhibit a broader range of variation of the porosity with the chorination temperature, most probably due to a better level of annealing of the structures. The porosity, however, depends sensitively on the density of the material (see Fig. 3.7). With the geometrical definition given above, the porosity of graphite results in a negligible value, as is confirmed from adsorption experiments. The simulations lead to small values of the porosity for both M1 and M2 materials for densities of the order of the density of graphite, and zero porosities for larger densities. The simulated M1 and M2 materials exhibit the same porosities for the same densities, confirming once more the weak dependence on the initial configuration of the $\mathrm{C}$ atoms and the more relevant dependence on the density of the material.

The gas adsorption capacity of a material is related with its specific surface area and its porosity. However, it has been shown both, from the experimental [24, 25] and theoretical [26] sides, that the adsorption capacity does not perfectly correlate with these two quantities but it depends on the size of the pores. For instance, it has been determined that the optimum pore size for hydrogen adsorption at room temperature is in the range of $7-11 \AA$ [27]. Thus, to fully characterize the porous structure of a material and its adsorption characteristics it is convenient to determine the pore size distribution function (PSD), that measures the total volume contained in pores of a given size. From the simulation side, PSD is a very demanding quantity to compute since large simulation cells are required to obtain meaningful distributions. The simulation cells considered in this work (of about $50 \AA$ wide) are sufficiently large to reproduce the distribution functions for pores smaller than about $20 \AA$. Larger pores are escarcely produced in these cells and, therefore, the distribution functions are not accurate for pores larger than about $20 \AA$. On the other hand, since the pores have not regular shapes and are interconnected among them, one has to introduce a model of pores to determine their size distribution. Based on our simulated structures we found appropriate to use a geometrical model of nonoverlapping spheres [4] to represent the pores. Figure 3.8 shows the pore size distribution function, PSD, of the CDCs obtained using $\mathrm{SiC}$ as the precursor, for several simulation temperatures. The CDCs produced at low temperatures $(T=350 \mathrm{~K})$ exhibit a relatively narrow distribution of pore sizes around an average value of about $8 \AA$. With increasing simulation temperature, the distribution becomes wider and the the 

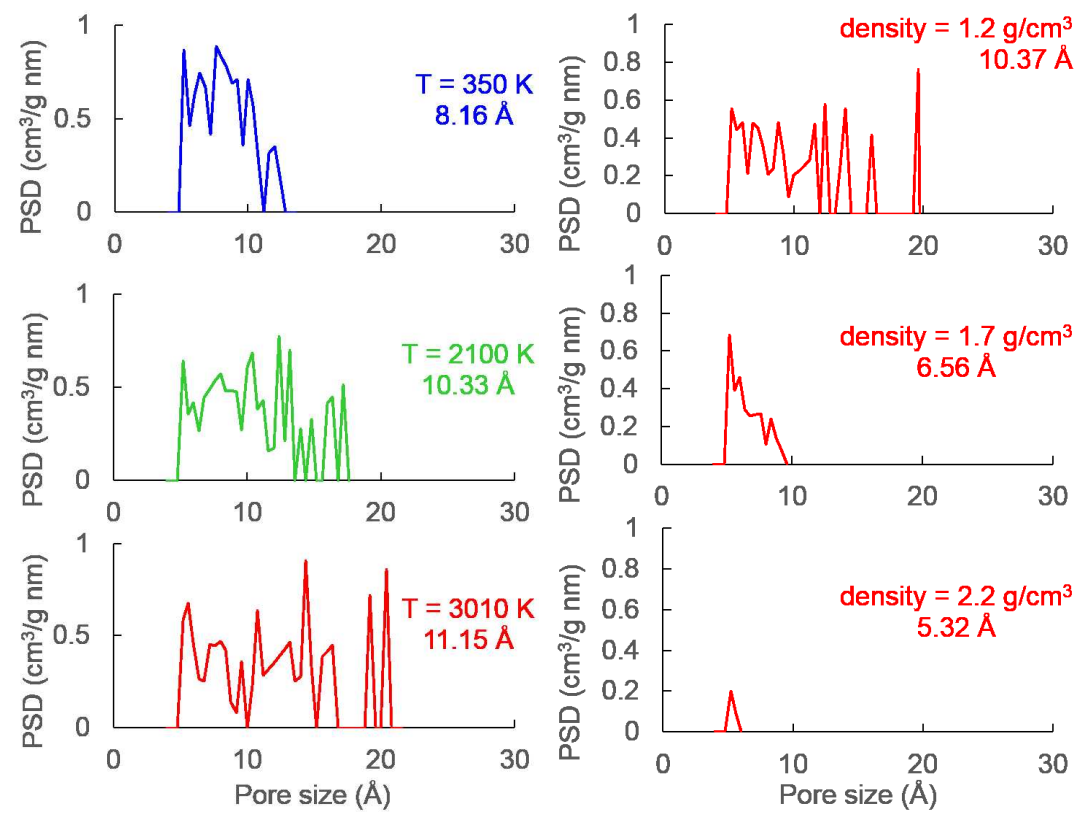

Fig. 3.8 Pore size distribution function, PSD of the CDCs obtained using $\mathrm{SiC}$ as the precursor, for several simulation temperatures (left panels). PSD of M2 materials of various densities produced at a temperature of $T=3010 \mathrm{~K}$ (right panels). Average pore size is indicated in each graph.

average pore size increases up to a value of about $11 \AA$ for $T=3010 \mathrm{~K}$. Although the maximum size of the pores (about $20 \AA$ ) is limited by the size of the simulation cell, this does not affect the general trends of the PSDs below that size. A similar behaviour has been obtained for the $\mathrm{CDCs}$ derived from $\mathrm{ZrC}$ as a precursor. The trends in the simulated PSD functions are in good agreement with the PSDs determined from experimental adsorption isotherms for CDCs produced from several precursor carbides [3].

On the other hand, similarly to the porosity, the PSD depends strongly on the density of the material. The PSD becomes narrower with increasing density and the size of the pores decreases. At approximately the density of graphite, the network of $\mathrm{C}$ atoms does not leave empty space and therefore the material does not contain pores any more (see Fig. 3.8 for M2 materials; a similar behaviour has been found for M1 materials).

\subsection{Conclusions}

We have performed atomistic molecular dynamics simulations to investigate the formation and structural characteristics of disordered carbon materials as a function of the $\mathrm{C}$ density, the initial carbon structure and the simulation temperature. Carbon 
materials ranging from CDCs porous carbons with densities of $0.77-0.96 \mathrm{~g} / \mathrm{cm}^{3}$ to amorphous carbons with densities of $2.8 \mathrm{~g} / \mathrm{cm}^{3}$ have been considered. The simulations have been designed to closely mimic the experimental process of formation of CDCs from metal carbides once the extraction of the metal has taken place and generalized for materials with different densities. Thus, the initial configurations for the NVT dynamical simulations were the structures of the $\mathrm{C}$ atoms in two carbides, $\mathrm{ZrC}$ an $\mathrm{SiC}$. Moreover, materials with different densities are generated using those two initial configurations but scaling the $\mathrm{C}-\mathrm{C}$ distances to produce the desired densities. Upon removal of the metal from the carbide, the collapse of the structure takes place very fast, in about a few ps, giving rise to a $\mathrm{C}$ network of $s p^{2} \mathrm{C}$ atoms. A porous structure of open interconnected pores appears; the pore walls are one atom thick graphitic-like layers interconnected among them. The level of graphitization of the pore walls is assessed in terms of the coordinations of the $\mathrm{C}$ atoms and the ring structure of the $\mathrm{C}$ network. For the CDCs and the low density $\mathrm{C}$ materials with densities below $1.7 \mathrm{~g} / \mathrm{cm}^{3}$, there is a prevalence of three-fold coordinated $\mathrm{C}$ atoms and hexagonal rings, confirming the graphitic-like structure of the pore walls. However, there is a non negligible number of two-fold coordinated $\mathrm{C}$ atoms, corresponding to the edges of the graphitic flakes, and heptagonal and octagonal rings that lead to open pores. With increasing temperature, the level of graphitizacion improves, although the edges and the heptagonal/octogonal defects do not disappear completely. At higher densities, of the order or higher than the density of graphite, there is still a prevalence of the coordination three, but four-fold coordinated $\mathrm{C}$ atoms appear and its percentage increases with increasing density. The carbon network loses progressively its graphitic character with the appearance of diamond-like tetrahedral structures that mark the transition towards amorphous carbons. Thus, the high density materials are formed by a combination of $s p^{2}$ and $s p^{3} \mathrm{C}$ atoms and the three dimensional $\mathrm{C}$ network does not enclose pores.

The specific surface area (SSA), the porosity, and the pore size distribution function (PSD) characterize the adsorption properties of a material. In agreement with experimental determinations, we found that the SSA of the simulated CDCs increases with increasing temperature, reaching values close to $2000 \mathrm{~m}^{2} / \mathrm{g}$. The porosity of the CDCs and the average size of the pores also increase with increasing temperature and the PSD becomes wider in close analogy with the experimental trends. However, with increasing carbon densities, the porosity drops dramatically and, as expected, for densities of the order and higher than the density of graphite the porosity vanishes.

In summary, we have found that the structure of the simulated carbon materials is not sensitive to the initial configuration of the $\mathrm{C}$ atoms. However, the structure is strongly dependent on the density, observing a transition from porous to amorphous structures at approximately the density of graphite. High temperatures favor higher level of graphitization in lower density, CDCs carbons.

Acknowledgements This work was supported by MINECO of Spain (Grant MAT2014-54378-R) and Junta de Castilla y León (Grant VA050U14). The authors thankfully acknowledge the facilities provided by Centro de Proceso de Datos - Parque Científico of the University of Valladolid. 


\section{References}

1. W.A. Mohun, in Proceedings of the 4th biennial conference on carbon, ed. by Y. Zhou (Pergamon Press, Oxford, 1960), pp. 443-453

2. Y. Gogotsi, A. Nikitin, H. Ye, W. Zhou, J.E. Fischer, B. Yi, H.C. Foley, M.W. Barsoum, Nat. Mater. 2(9), 591 (2003). DOI 10.1038/nmat957

3. G. Yushin, R. Dash, J. Jagiello, J. Fischer, Y. Gogotsi, Adv. Funct. Mater. 16(17), 2288 (2006). DOI 10.1002/adfm.200500830

4. M.J. López, I. Cabria, J.A. Alonso, J. Chem. Phys. 135(10), 104706 (2011). DOI $10.1063 / 1.3633690$

5. J.A. Alonso, I. Cabria, M.J. López, J. Mater. Res. 28(4), 589 (2013). DOI 10.1557/jmr.2012.370

6. C. de Tomás, I. Suarez-Martinez, F. Vallejos-Burgos, M.J. López, K. Kaneko, N.A. Marks, Carbon 119, 1 (2017). DOI 10.1016/j.carbon.2017.04.004

7. J.C. Angus, C.C. Hayman, Science 241(4868), 913 (1988). DOI $10.1126 /$ science. 241.4868 .913

8. F.P. Bundy, W.A. Bassett, M.S. Weathers, R.J. Hemley, H.U. Mao, A.F. Goncharov, Carbon 34(2), 141 (1996). DOI 10.1016/0008-6223(96)00170-4

9. J. Narayan, A. Bhaumik, J. Appl. Phys. 118(21), 215303 (2015). DOI 10.1063/1.4936595

10. B. Stenhouse, P.J. Grout, N.H. March, J. Wenzel, Phil. Mag. 36(1), 129 (1977). DOI 10.1080/00318087708244453. Reprinted in Ref. [28].

11. B.J. Stenhouse, P.J. Grout, J. Non-Cryst. Sol. 27(2), 247 (1978). DOI 10.1016/00223093(78) $90127-8$

12. D.F.R. Mildner, J. Carpenter, J. Non-Cryst. Solids 47(3), 391 (1982). DOI 10.1016/00223093(82)90215-0

13. C.A. Majid, J. Non-Cryst. Solids 57(1), 137 (1983). DOI 10.1016/0022-3093(83)90416-7

14. T. Noda, M. Inagaki, Bull. Chem. Soc. Jap. 37(10), 1534 (1964). DOI 10.1246/bcsj.37.1534

15. E. Fitzer, K. Kochling, H.P. Boehm, H. Marsh, Pure Appl. Chem. 67, 473 (1995). DOI 10.1351/pac199567030473. (IUPAC Recommendations 1995)

16. V.L. Deringer, G. Csányi, Phys. Rev. B 95, 094203 (2017). DOI 10.1103/PhysRevB.95.094203

17. C. de Tomas, I. Suarez-Martinez, N.A. Marks, Carbon 109, 681 (2016). DOI 10.1016/j.carbon.2016.08.024

18. J. Tersoff, Phys. Rev. B 37, 6991 (1988). DOI 10.1103/PhysRevB.37.6991

19. J. Tersoff, Phys. Rev. Lett. 61, 2879 (1988). DOI 10.1103/PhysRevLett.61.2879

20. Marcos, P. A., Alonso, J. A., Rubio, A., López, M. J., Eur. Phys. J. D 6(2), 221 (1999). DOI $10.1007 / \mathrm{s} 100530050304$

21. K. Nordlund, J. Keinonen, T. Mattila, Phys. Rev. Lett. 77, 699 (1996). DOI 10.1103/PhysRevLett.77.699

22. M.J. López, I. Cabria, N.H. March, J.A. Alonso, Carbon 43(7), 1371 (2005). DOI 10.1016/j.carbon.2005.01.006

23. R.K. Dash, G. Yushin, Y. Gogotsi, Micropor. Mesopor. Mat. 86(1), 50 (2005). DOI 10.1016/j.micromeso.2005.05.047

24. A. Linares-Solano, M. Jordá-Beneyto, D.L.C.M. Kunowsky, F. Suárez-García, D. CazorlaAmorós, in Carbon Materials: Theory and Practice, ed. by A. Terzyk, P. Gauden, P. Kowalczyk (Research Signpost, Kerala, India, 2008), pp. 245-281

25. Y. Gogotsi, R.K. Dash, G. Yushin, T. Yildirim, G. Laudisio, J.E. Fischer, J. Am. Chem. Soc. 127(46), 16006 (2005). DOI 10.1021/ja0550529

26. I. Cabria, M.J. López, J.A. Alonso, Carbon 45(13), 2649 (2007). DOI 10.1016/j.carbon.2007.08.003

27. I. Cabria, M.J. López, J.A. Alonso, Int. J. Hydrogen Energy 36(17), 10748 (2011). DOI 10.1016/j.ijhydene.2011.05.125. International Conference on Hydrogen Production (ICH2P)2010

28. N.H. March, G.G.N. Angilella (eds.), Many-body theory of molecules, clusters, and condensed phases (World Scientific, Singapore, 2009) 